\title{
ELECTRA-DTA: A New Compound-protein Binding Affinity Prediction Model based on the Contextualized Sequence Encoding
}

\section{junjie wang}

Nanjing Medical University https://orcid.org/0000-0003-0732-051X

\section{NaiFeng Wen}

Dalian Minzu University: Dalian Nationalities University https://orcid.org/0000-0003-2725-8645

\section{Chunyu Wang}

Harbin Institute of Technology https://orcid.org/0000-0002-2965-9920

Lingling Zhao ( $\nabla$ zhaoll@hit.edu.cn )

Harbin Institute of Technology

\section{Liang Cheng}

Harbin Medical University https://orcid.org/0000-0002-6665-6710

\section{Research article}

Keywords: deep learning, representative learning, drug-target affinity prediction, ELECTRA

Posted Date: October 5th, 2021

DOl: https://doi.org/10.21203/rs.3.rs-934203/v1

License: (c) (i) This work is licensed under a Creative Commons Attribution 4.0 International License. Read Full License

Version of Record: A version of this preprint was published at Journal of Cheminformatics on March 15th, 2022. See the published version at https://doi.org/10.1186/s13321-022-00591-x. 


\title{
RESEARCH
}

\section{ELECTRA-DTA: A new compound-protein binding affinity prediction model based on the contextualized sequence encoding}

\author{
Wang Junjie ${ }^{1}$, Wen NaiFeng ${ }^{2}$, Wang Chunyu ${ }^{3}$, Zhao Lingling ${ }^{3 *}$ \\ and Cheng Liang ${ }^{4}$
}

${ }^{*}$ Correspondence:

zhaoll@hit.edu.cn

${ }^{3}$ Faculty of Computing, Harbin Institute of Technology, Harbin, P. R. China

Full list of author information is available at the end of the article

\begin{abstract}
Motivation: Drug-target binding affinity (DTA) reflects the strength of the drug-target interaction; therefore, predicting the DTA can considerably benefit drug discovery by narrowing the search space and pruning drug-target (DT) pairs with low binding affinity scores. Representation learning using deep neural networks has achieved promising performance compared with traditional machine learning methods; hence, extensive research efforts have been made in learning the feature representation of proteins and compounds. However, such feature representation learning relies on a large-scale labelled dataset, which is not always available.

Results: We present an end-to-end deep learning framework, ELECTRA-DTA, to predict the binding affinity of drug-target pairs. This framework incorporates an unsupervised learning mechanism to train two ELECTRA-based contextual embedding models, one for protein amino acids and the other for compound SMILES string encoding. In addition, ELECTRA-DTA leverages a squeeze-and-excitation (SE) convolutional neural network block stacked over three fully connected layers to further capture the sequential and spatial features of the protein sequence and SMILES for the DTA regression task. Experimental evaluations show that ELECTRA-DTA outperforms various state-of-the-art DTA prediction models, especially with the challenging, interaction-sparse BindingDB dataset. In target selection and drug repurposing for COVID-19, ELECTRA-DTA also offers competitive performance, suggesting its potential in speeding drug discovery and generalizability for other compound- or protein-related computational tasks.
\end{abstract}

Keywords: deep learning; representative learning; drug-target affinity prediction; ELECTRA

\section{Introduction}

Drug discovery and development are laborious, time-consuming, expensive and challenging processes. One of the most important steps in developing a new drug or repurposing existing drugs is target identification and validation. A direct bruteforce search is unrealistic because of the very large number of drug-like compounds and possible drug targets. Given current advances in computational methods and techniques, especially the application of machine learning in chemical and biological research fields, computer-aided methods may be a great opportunity to shorten the drug discovery process by significantly narrowing the search space. 
Apart from the prediction of binary interaction relationships between compoundprotein pairs, another crucial factor in candidate screening, the prediction of the binding affinity of compound-protein pairs, also called drug-target affinity (DTA), remains a challenge in drug discovery. Laboratory experiments conducted to measure the affinity value for a large-scale drug-target pool remain time consuming and expensive. Hence, computational methods to perform binding affinity prediction have received increasing attention in recent years, and much effort has been made to accurately quantify the strength of binding for compound-protein pairs based on machine learning or deep learning. Gradient boosting machines are used in quantitative structure-activity relationship studies for regression and classification problems. SimBoost [1] employs gradient boosting machines with novel feature engineering to extract new features from drugs, targets and drug-target pairs in training datasets, and then these features are used as inputs to models to predict the binding affinity for unknown pairs. Regularized least-square (RLS) is another efficient model with various applications. The KronRLS [2] model amends RLS with the Kronecker products of drug-drug and protein-protein interactions to speed up model training for DTA prediction and has achieved promising performance. The Kronecker product part of the KronRLS is a similarity-based method in which any similarity measure could be used.

Recently, inspired by successful applications in diverse research fields, deep learning approaches have also been intensively used in bioinformatics and cheminformatics, especially in drug discovery. The first deep learning-based DTA prediction model was DeepDTA [3], which uses simplified molecular input line entry system (SMILES), a one-dimensional representation of the drug compound chemical structure, as drug features, while the protein amino acid sequences are used to represent protein features. Furthermore, the drug SMILESs are labelled as encoded integer vectors and as protein sequences. The DeepDTA model uses a CNN with three 1D convolutional layers with pooling for drug embedding to learn latent features for each drug and an identical CNN for protein embedding. Then, each pair of drug-target feature vectors is concatenated and fed into fully connected layers for training and prediction. Another novel deep learning model for DTA is DeepAffinity [4], which represents drugs with SMILESs and proteins with structural property sequences. Because of the detailed structural information and higher resolution of sequences, DeepAffinity benefits DTA regression tasks. The drug SMILESs and protein structural sequences are both encoded into embedding representations by a recurrent neural network (RNN) auto-encoder model named seq2seq [5]. The seq2seq model maps raw sequences into vectors that are learned in an unsupervised fashion to capture dependencies in sequences of SMILESs or protein residues.

The aforementioned DTA models mainly focus on developing diverse neural network architectures to learn the hierarchical feature representations on given, known CP pairs with binding affinity values in a non-handcraft manner. These existing models usually take predefined compound molecular and protein descriptors as input features, especially SMILES strings and protein residue sequences. To be fed into deep networks such as CNNs or LSTMs, the raw sequence or SMILES strings are encoded by one-hot vectors, physicochemical property-aware encoding or static embeddings. Such mechanisms deploy fixed representations, which makes 
the model characterize each amino acid or atom independently but do not consider the contextual information and accordingly do not highlight those that are critical to the whole protein or molecular compound. Applicable experimental structures of protein complexes are abundant, but the number of available labelled drug-target pairs remains limited. To address the issue of insufficient labelled data, unsupervised encoding methods have recently been considered for proteins or compounds. The idea behind this approach is to use protein sequences and compound SMILESs or fingerprints as a codified language for human experts with limited words and grammar. Similar to a linguist who extracts hidden knowledge from sentences of a natural language, the structure and function of chemical compounds and protein sequences can be processed deliberately to build novel solutions, such as DTA predictions, based on the level of understanding. State-of-the-art representation models for NLP, such as BERT [6], have greatly improved downstream NLP tasks due to their advantages. The recent transformer-based ELECTRA [7] model uses training data efficiently and shows better performance than BERT. ELECTRA employs a generative-discriminative model to make better use of training data; a generator model replaces some tokens in the corpus, and a co-trained discriminative model detects these replacements. Because the corpus is well used in this fashion, ELECTRA is more efficient than BERT with comparable model sizes, especially small models. This paper proposes ELECTRA-based encoders for molecular SMILESs and protein sequences separately and accordingly yields a novel DTA prediction framework incorporating an ELECTRA encoding layer, a CNN network and a regression block. The proposed framework enhances the feature representation capability as follows. First, the ELECTRA encoding layers used to represent each molecule can take advantage of existing chemical structure knowledge by pretraining on the PubChem database to extract useful chemical information, and a similar prompt occurs for the amino acid representation. Finally, the ELECTRA encoding provides the contextrelated representation for protein sequences and compound SMILESs, which can characterize the diversity of each atom or amino acid in a variety of sequences or strings.

In summary, our method consists of two major building blocks: one for feature representation and another for regression prediction. The first block, using two pretrained ELECTRA models, extracts local context information from drug SMILES strings and raw protein sequences separately. Then, the learned representations for both drugs and targets are passed into the second block, which uses a fully connected neural network to predict the binding affinity as a regression task. Our framework needs neither expert knowledge nor the 3D structure of the targets, so it is more convenient than existing frameworks. Additionally, the proposed framework takes advantage of the local chemical context information of atoms in drugs or amino acids in proteins, which differentiates ELECTRA-DTA from existing deep learning models. The main contributions of this paper are as follows:

1 We adopt ELECTRA, a state-of-the-art NLP model, to extract feature representations from raw sequence data, taking advantage of the local context information.

2 We leverage a squeeze-and-excitation convolution neural network block stacked over three fully connected layers to capture the sequential and spatial features of the protein sequence and SMILES for the DTA regression task. 
3 We applied the ELECTRA-DTA model for target selection and drug repur- ${ }_{105}$ posing for COVID-19 and again obtained competitive performance. $\quad 106$

\section{Methods}

In this section, we first summarize the whole model. Then, we introduce the input ${ }_{108}$ representation for compounds and proteins. After that, we show the training for ${ }_{109}$ ELECTRA and the representation tensor of compounds and proteins. Finally, we ${ }_{110}$ provide the details of our training and prediction model .

Overview of the ELECTRA-DTA model

Figure 1 shows the overview of ELECTRA-DTA. It takes the SMILES strings of compounds and the amino acid sequences of proteins as the input. By incorporating a pre-trained ELECTRA model, ELECTRA-DTA encodes these sequences into feature tensors as internal representations. Then, the model exploits a CNN network to learn from known drug-protein pairs in a supervised manner. Finally, it outputs the binding affinity for new pairs. The design of ELECTRA-DTA includes three main steps:

figure1.jpg

Figure 1 Overview of ELECTRA-DTA

1 The training of two ELECTRA models to encode all amino acids for protein ${ }_{120}$ sequences and characters in SMILESs for compounds separately; ${ }_{121}$

2 The encoding of the whole sequence of compounds and proteins as feature ${ }_{122}$ tensors;

3 Model training and predicting the binding affinity with the proposed deep ${ }^{124}$ neural network.

Pretrained ELECTRA models for encoding protein sequences and compound SMILES strings

To encode the SMILESs/amino acid sequences, existing deep learning approaches $\quad{ }_{128}$ such as DeepDTA and DeepCDA use label/one-hot encoding to represent each ${ }_{129}$ symbol in the SMILES/amino acid sequence. However, label/one-hot encoding often $\quad{ }_{130}$ neglects the context of the symbol and thus cannot reveal the functionality of the ${ }_{131}$ symbol within the context.

Construction of the compound SMILES corpora We need to train the ELECTRA ${ }_{133}$ model with an appropriate corpus for a specific task in advance to obtain an en- ${ }_{134}$ coding layer for input sequences. Compound SMILES are linguistic constructs with ${ }_{135}$ a simple vocabulary (only atoms and chemical bond symbols) and a few grammar ${ }_{136}$ rules. Similar to natural language processing, compound SMILES strings are anal- ${ }_{137}$ ogous to sentences, where each atom and bond symbol is a word (token). A corpus ${ }_{138}$ can then be naturally composed by collecting numerous compounds. For exam- ${ }_{139}$ ple, the SMILES string " $\mathrm{CC}(=) \mathrm{OC} 1=\mathrm{C}$ " can be listed as a sentence composed of ${ }_{140}$ the tokens 'C','C','(','=,','),'O','C','1','=','C'. The corpus we constructed contains ${ }_{141}$ 
all canonical SMILES extracted from PubChem $[8,9,10]$ with a total number of ${ }_{142}$ 1114424 .

To ensure the consistency of all SMILES from different sources, we use Open ${ }_{144}$ Babel [11] v3.1.0 to convert all SMILES strings in the corpora into a canonical ${ }_{145}$ format, which is also applied to SMILES in all datasets. Detailed information on ${ }_{146}$ the SMILES corpora we constructed is listed in Table 1.

Table 1 Statistics of the compound SMILES corpora

\begin{tabular}{llll}
\hline No. of corpus & $\begin{array}{l}\text { Average length of the } \\
\text { corpus }\end{array}$ & $\begin{array}{l}\text { Minimum length of } \\
\text { the corpus }\end{array}$ & No. of vocabulary \\
\hline 1114424 & 47 & 3 & 72 \\
\hline
\end{tabular}

Construction of the protein FASTA sequence corpora Similar to the compound ${ }_{148}$ SMILES, a 1-mer schema is exploited to extract words from the protein FASTA 149 sequences and create a protein sequence corpora; in this way, each residue is a sin- ${ }_{150}$ gle word. We utilized the Swiss-Prot dataset from the UniProt knowledgebase [12] ${ }_{151}$ (UniProtKB), which included 563,552 manually annotated and reviewed protein ${ }_{152}$ sequences, to collect the proteins. UniProtKB/Swiss-Prot is a high-quality anno- ${ }_{153}$ tated and non-redundant protein sequence database. The detailed information on ${ }^{154}$ the protein sequence corpora we constructed is listed in Table 2.

Table 2 Statistics of protein sequence corpora

\begin{tabular}{llll}
\hline No. of corpus & $\begin{array}{l}\text { Average length of the } \\
\text { corpus }\end{array}$ & $\begin{array}{l}\text { Minimum length of } \\
\text { the corpus }\end{array}$ & No. of vocabulary \\
\hline 1868198 & 382 & 2 & 30 \\
\hline
\end{tabular}

Pretraining the ELECTRA models Our method exploits two separate ELECTRA models to separately encode the SMILESs and amino acid sequences into fixed dimensional vectors. In an effort to avoid confusion and awkward phrasing, we describe the procedure of training the ELECTRA model for the SMILESs. The procedure for the pre-trained ELECTRA model for the amino acids sequences is similar.

ELECTRA exploits two Transformer encoders as the base structure: one acts as a generator network, and the other acts as a discriminator network. The generator is typically a small, masked language model that produces an output distribution over the tokens. The tokens from a SMILES string are first masked, and some are replaced with a mask symbol [MASK] with a constant probability. Then, the masked tokens are fed into the generator for joint pre-training with the discriminator. The generator network first learns from the masked tokens and then fills the missing tokens with predicted values, but the predicted value may not be the same as the original value. However, the jointly trained discriminator network learns to resolve whether each token is the same as the original one. It uses another Transformer encoder to extract the contextual information as embedding representations, which are used to determine the probability of replacing the tokens, as shown in figure $2 . \quad{ }^{173}$ After pre-training with SMILES strings, we obtain an ELECTRA-M model as an ${ }_{174}$ encoder that can encode each SMILES into a feature vector in downstream tasks. ${ }^{175}$ Similarly, we obtain an ELECTRA-P model pre-trained with protein sequences that ${ }_{176}$ also encode each protein sequence into a feature vector. 


$$
\text { [scale }=0.5] \text { figure2.jpg }
$$

Figure 2 The principle of ELECTRA pre-training

Input representation

Protein sequence and compound SMILES are fed into the entire framework as in- ${ }_{179}$ put; because the SMILES strings and protein sequences are of different lengths, we ${ }_{180}$ truncate them to fixed lengths for effective representation. As in [3], we also choose ${ }_{181}$ fixed lengths of 100 for SMILES and 1000 for protein sequences for the bench- ${ }_{182}$ mark of datasets. We chose these maximum lengths based on the distributions of ${ }_{183}$ the datasets so that the maximum lengths cover at least $80 \%$ of the proteins and ${ }^{184}$ compounds in the datasets. Longer sequences are truncated to these lengths, while ${ }_{185}$ shorter sequences are padded with zeros to the fixed lengths. ${ }_{186}$

Compound SMILES and protein sequence embedding

With the trained ELECTRA-M and ELECTRA-P models, the input compound SMILES strings and protein sequences are embedded into tensors separately. For individual compounds, the sequence of tokens from their SMILES strings, which represents atoms or structure indicators, is fed into the trained ELECTRA-M model to yield a compound encoding. Specifically, each token, which is one character, is converted to a vector of length $W_{c}$ by ELECTRA-M, and then a sequence of $N_{c}$ tokens is converted to a sequence of $N_{c}$ vectors that are finally concatenated into a $W_{c} \times N_{c}$ tensor as the compound representation. In the same way, one protein residue token is encoded into a vector of length $W_{p}$ by ELECTRA-P, and a protein sequence of length $N_{p}$ vectors that are concatenated into a $W_{p} \times N_{p}$ tensor, as shown in figure 3 .

$$
[\text { scale }=0.5] \text { figure3.jpg }
$$

Figure 3 Compound SMILES and protein sequence embedding

\section{ELECTRA-DTA framework}

In this work, the ELECTRA-DTA model incorporates two identical feature extractor networks and one regression network, as shown in figure 4. Each feature extractor network is a typical convolutional neural network equipped with two layers of stacked squeeze-and-excitation (SE) blocks in addition to one global max pooling filter layer. The feature extractor network takes the compound or protein tensor as input to learn latent features during the supervised training process and then produces feature vectors as a representation. Two feature vectors are then concatenated into a single vector, which is fed into the regression network for the prediction.

\section{[]figure4.jpg}

Figure 4 Architecture of the ELECTRA-DTA model 
[]figure5.jpg

Figure 5 Structure of the squeeze-and-excitation block

Inside the feature extractor network, the main blocks are two SE block layers. ${ }_{209}$ These are recently proposed CNN units that can be stacked for extremely effective ${ }_{210}$ generalization across different datasets, especially image processing. The SE block ${ }^{211}$ improves the joint encoding of image spatial and channel information by remov- ${ }_{212}$ ing the spatial dependency with global average pooling to learn a channel-specific ${ }^{213}$ descriptor. This block is capable of feature recalibration by using global informa- ${ }^{214}$ tion to selectively emphasize informative features over the others. The SE block of ${ }_{215}$ the ELECTRA-DTA model is shown in figure 5. We use one-dimensional convolu- ${ }_{216}$ tion to project the input compound or protein tensor $X \in \mathbb{R}^{T \times 256}$ into the feature ${ }^{217}$ maps $U \in \mathbb{R}^{T \times C}$ to perform feature recalibration. This one-dimensional convolu- ${ }^{218}$ tion is in the feature direction. Therefore, these feature maps $U$ are first squeezed $\quad{ }_{219}$ and produce a channel (or feature) descriptor by global average pooling in the fea- 220 ture direction. The interaction information is accumulated in this descriptor. The ${ }_{221}$ squeezing operation is followed by an excitation operation with a fully connected 222 layer and ReLU activation, which produces modulation weights from the features ${ }^{223}$ by a simple self-gating mechanism. The output of the SE block is in the form of the ${ }^{224}$ feature maps $U$ scaled by these modulation weights. The stacked SE block further ${ }^{225}$ enlarges this interaction information between features. 226

The regression network contains two tandem parts, as shown in figure 4 . The first ${ }^{227}$ tandem part consists of a stack of fully connected layers with a simple gating mecha- ${ }_{228}^{228}$ nism. The second tandem part has four fully connected layers of different sizes. The ${ }_{229}$ simple gating mechanism in the first part is adopted from [13] to better regulate 230 information flow in consecutive training epochs. Each layer consists of two gates ${ }^{231}$ called a Carry and a Transform that separate information flow to two streams - one 232 modified, the other untouched - to the next iteration. Finally, all information flows 233 through four fully connected layers and then produces the predicted affinity score. ${ }^{234}$

\section{Model validation}

Datasets

This study evaluated ELECTRA-DTA using three common benchmark datasets: ${ }^{237}$ the KIBA dataset [14], the Davis dataset [15], and the BindingDB dataset [16]. The ${ }_{238}$ Davis dataset consists of 442 proteins and 68 compounds forming 30,056 DT pairs, ${ }^{239}$ while the KIBA dataset contains 229 proteins and 2111 compounds forming 118,254 240 DT pairs. BindingDB is a web-accessible public dataset, and the filtered version is ${ }_{241}$ exploited in this study to maintain consistency with a previous study [4]. Table $3{ }^{242}$ provides the statistics of these datasets.

However, the versions of these datasets used here share one crucial problem: ${ }^{244}$ the same DT sequence has different or duplicate affinity values. We use the ${ }^{245}$ Davis dataset as an example to illustrate this phenomenon. The ABL1 proto- ${ }_{246}$ oncogene encodes a cytoplasmic and nuclear protein tyrosine kinase that has ${ }_{247}$ been implicated in the processes of cell differentiation, cell division, cell adhesion, ${ }^{248}$ and stress response [17]. The ABL1(Y253F) mutant is a Gleevec-resistant form ${ }^{249}$ 
Table 3 The detailed statistics of the datasets, containing the number of proteins, compounds, interactions and average number of interactions per protein and per compound.

\begin{tabular}{llllllll} 
& $\begin{array}{l}\text { No. Pro- } \\
\text { teins }\end{array}$ & $\begin{array}{l}\text { No. } \\
\text { Com- } \\
\text { pounds }\end{array}$ & $\begin{array}{l}\text { No. In- } \\
\text { teraction }\end{array}$ & $\begin{array}{l}\text { No. } \\
\text { interac- } \\
\text { tions per } \\
\text { protein }\end{array}$ & $\begin{array}{l}\text { No. } \\
\text { inter- } \\
\text { actions } \\
\text { per com- } \\
\text { pound }\end{array}$ & $\begin{array}{l}\text { No. in- } \\
\text { active } \\
\text { interac- } \\
\text { tions }\end{array}$ & $\begin{array}{l}\text { No. non- } \\
\text { inactive } \\
\text { interac- } \\
\text { tions }\end{array}$ \\
\hline Davis & 442 & 68 & 30056 & 68 & 442 & 2502 & 27554 \\
KIBA & 229 & 2111 & 118254 & 516 & 56 & 24828 & 93426 \\
BindingDB & 1620 & 87461 & 144525 & 89 & 2 & 47608 & 96917 \\
\hline
\end{tabular}

of ABL1. ABL1 and ABL1(Y253F) share same amino acid sequence, and their 250 binding affinity values for the drug fedratinib (Pubchem ID: 16722836, SMILES: ${ }^{251}$ $\mathrm{CC} 1=\mathrm{CN}=\mathrm{C}(\mathrm{N}=\mathrm{C} 1 \mathrm{NC} 2=\mathrm{CC}(=\mathrm{CC}=\mathrm{C} 2) \mathrm{S}(=\mathrm{O})(=\mathrm{O}) \mathrm{NC}(\mathrm{C})(\mathrm{C}) \mathrm{C}) \mathrm{NC} 3=\mathrm{CC}=\mathrm{C}\left(\mathrm{C}={ }_{252}\right.$ C3)OCCN4CCCC4) are 6.7447 and 7.2518, respectively. The MET(Y1235D) re- ${ }^{253}$ combinant human protein (956-end) was expressed in insect cells. MET receptor ${ }^{254}$ tyrosine kinase is a proto-oncogene that encodes a transmembrane growth factor 255 receptor that is a heterodimer of two disulfide-linked chains of $50 \mathrm{kDa}$ (alpha) ${ }_{256}$ and $145 \mathrm{kDa}$ (beta) [18]. They also share the same amino acid sequence, but their ${ }_{257}^{257}$ binding affinity values for the drug Bms-345541 (PubChem ID: 9926054, SMILES: 258 $\mathrm{CC} 1=\mathrm{CC} 2=\mathrm{C}(\mathrm{C}=\mathrm{C} 1) \mathrm{N}=\mathrm{C}(\mathrm{C} 3=\mathrm{NC}=\mathrm{C}(\mathrm{N} 23) \mathrm{C}) \mathrm{NCCN} . \mathrm{Cl})$ are both 5.0. 259

Duplicated samples may harm the effectiveness of the deep learning DTA model, $\quad 260$ while sample inputs (DT pairs) with different labels (binding affinity values) may ${ }_{261}$ be detrimental to training the deep learning DTA model. We thus removed these ${ }_{262}$ samples from the original datasets. Table [?] describes the details of the refined ${ }^{263}$ datasets.

Table 4 The detailed statistics of the refined datasets, containing the number of proteins, compounds, interactions and average number of interactions per protein and per compound.

\begin{tabular}{llllllll} 
& $\begin{array}{l}\text { No. Pro- } \\
\text { teins }\end{array}$ & $\begin{array}{l}\text { No. } \\
\text { Com- } \\
\text { pounds }\end{array}$ & $\begin{array}{l}\text { No. In- } \\
\text { teraction }\end{array}$ & $\begin{array}{l}\text { No. } \\
\text { interac- } \\
\text { tions per } \\
\text { protein }\end{array}$ & $\begin{array}{l}\text { No. } \\
\text { inter- } \\
\text { actions } \\
\text { per com- } \\
\text { pound }\end{array}$ & $\begin{array}{l}\text { No. in- } \\
\text { active } \\
\text { interac- } \\
\text { tions }\end{array}$ & $\begin{array}{l}\text { No. non- } \\
\text { inactive } \\
\text { interac- } \\
\text { tions }\end{array}$ \\
\hline Davis & 361 & 68 & 24548 & 68 & 61 & 1649 & 22899 \\
KIBA & 229 & 2052 & 117184 & 511.720 & 57.107 & 24543 & 92641 \\
BindingDB & 1615 & 129109 & 144525 & 79.944 & 1.541 & 41487 & 87622 \\
\hline
\end{tabular}

It can be observed that these datasets differ significantly in terms of the number ${ }_{265}$ of interactions per protein or compound. For the Davis and KIBA datasets, the ${ }_{26}$ average number of interactions per protein and compound is higher than 50, whereas 267 BindingDB contains much fewer interactions for both compounds and proteins, 268 suggesting that the connections between compounds and proteins are sparse, which 269 would make the training of the prediction models challenging. In addition, all of ${ }_{270}$ these datasets have skewed distributions. ${ }_{271}$

Evaluation metrics $\quad{ }_{272}$

The performance of all models was measured by the concordance index [19] (CI), ${ }^{273}$ the mean squared error (MSE), Pearson correlation coefficient, the $r_{m}^{2}$ index $[20,21] \quad{ }_{274}$ 
and the area under precision recall (AUPR) curve score. CI indicates the ranking ${ }_{275}$ performance of the models and can be calculated by equations (1).

$$
\mathrm{CI}=\frac{1}{Z} \sum_{\delta_{x}>\delta_{y}} h\left(b_{x}-b_{y}\right), \quad \text { where } h(m)= \begin{cases}1, & \text { if } m>0 \\ 0.5, & \text { if } m=0 \\ 0, & \text { if } m<0\end{cases}
$$

The $r_{m}^{2}$ index defines the possibility of an acceptable model and is calculated by equation (2), where $r_{0}^{2}$ and $r^{2}$ are the squared correlation coefficients with and without intercepts, respectively.

$$
r_{m}^{2}=r^{2} *\left(1-\sqrt{r^{2}-r_{0}^{2}}\right)
$$

Generally, a model with an $r_{m}^{2}$ index greater than 0.5 on a test set can be consid- ${ }_{277}$ ered acceptable. The AUPR curve score is generally adopted for binary prediction; ${ }_{278}$ therein, we converted the regressions on these datasets into their binary forms by ${ }_{279}$ thresholding similarly to DeepDTA. The Pearson correlation (R) is a metric that ${ }_{280}$ measures the correlation between two continuous variables. ${ }^{281}$

There are three variant ELECTRA models: ELECTRA small, with 14M param- ${ }^{282}$ eters, ELECTRA base, with 110M parameters, and ELECTRA large, with 335M ${ }_{283}$ parameters. Due to limited computing resources, we only pretrain two ELECTRA ${ }^{284}$ small models, one for compounds and another for proteins. We use code from sim- ${ }^{285}$ pletransformers to train these two ELECTRA small models. Unlike with the NLP ${ }_{286}$ ELECTRA models, we use the atom tokenization method for the compound SMI- ${ }^{287}$ LESs and protein amino acid sequences. The pre-training task of the ELECTRA ${ }_{288}$ small models takes 6 days with a batch size of 256, a learning rate of $1 \mathrm{e}-5$, and ${ }_{289}$ an epoch of 100 by using 8 NVIDIA GeForce RTX 2080 Ti GPUs. The feature 290 extraction block comprises 2 convolutional layers, one with 256 and the other with 291 512 channels. All convolutional layers apply 3 convolution kernels and are activated 292 through rectified linear unit (ReLU) activation function. The regression block has ${ }^{293}$ 4 FC layers with 1024, 1024, 512, and 1 node. Each of the first two FC layers is ${ }^{294}$ followed by a dropout layer with a rate of 0.4. These $4 \mathrm{FC}$ layers also use ReLU as 295 the activation function. The Adam optimizer was used to train the parameters of ${ }_{296}$ the feature extraction block and regression block with the default learning rate of ${ }_{297}$ 0.001 for each DTA dataset. ${ }_{298}$

Results

Results of predictive performance of random splitting settings Four baseline approaches were selected for comparison with the proposed ELECTRA- 301 DTA, including two classical machine learning-based prediction models, KronRLS 302 and SimBoost, and two state-of-the-art deep learning-based models, DeepDTA and ${ }_{303}$ DeepCDA [22], when using the original datasets. It should be noted that to avoid 304 potential errors from the implementation and training of these models, the results 305 of the baseline approaches reported in the following tables are taken directly from 306 their original publications. As the authors for DeepCDA did not provide details ${ }_{307}$ 
on the hyperparameters of the network, we were unable to reproduce their meth- 308 ods. Therefore, we compared our method with another state-of-the-art method: 309 AttentionDTA [23].

Given the output of ELECTRA layer layer $_{k}$, we used two different input settings for the feature extractor network:

1 ELECTRA-DTA-AVG: Average of all token vectors from 12 Transformer layers.

2 ELECTRA-DTA-LAST: Vector representation of the last Transformer layer. ${ }_{315}$

To quantify the prediction performance in an unbiased and consistent manner, we 316 carried out 5-fold cross-validation similar to that used for DeepDTA, DeepCDA and 317 SimBoost; that is, we split the data arbitrarily into six equivalent parts in which one 318 part is selected as the independent test set. Any one of the remaining parts is used 319 as the validation set and the others as the training set. The purpose of this division 320 is to determine the hyper-parameters via 5-fold cross validation. Tables 5, 6 and 7321 report the results of all the evaluated models and their average CI, MSE, R, $r_{m}^{2}{ }_{322}$ and AUPR curve scores with the original and refined KIBA, Davis and BindingDB ${ }_{323}$ datasets, respectively.

Table 5 Comparison of all baseline approaches and ELECTRA-DTA on the KIBA datasets

\begin{tabular}{|c|c|c|c|c|c|c|}
\hline Dataset & Model & $\mathrm{Cl}$ & MSE & $\mathrm{R}$ & $r_{m}^{2}$ & AUPR \\
\hline \multirow{7}{*}{$\begin{array}{c}\text { Original } \\
\text { KIBA } \\
\text { Dataset }\end{array}$} & KronRLS & 0.782 & 0.411 & - & 0.342 & 0.635 \\
\hline & SimBoost & 0.836 & 0.222 & - & 0.629 & 0.760 \\
\hline & DeepDTA & 0.863 & 0.194 & 0.848 & 0.673 & 0.788 \\
\hline & WideDTA & 0.875 & 0.179 & 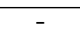 & - & - \\
\hline & DeepCDA & $0.889(0.002)$ & 0.176 & 0.855 & $0.682(0.008)$ & $0.812(0.005)$ \\
\hline & $\begin{array}{l}\text { ELECTRA- } \\
\text { DTA-AVG }\end{array}$ & $0.889(0.003)$ & 0.162 & 0.879 & $0.727(0.004)$ & $0.795(0.006)$ \\
\hline & $\begin{array}{l}\text { ELECTRA- } \\
\text { DTA- } \\
\text { LAST }\end{array}$ & $0.886(0.002)$ & 0.172 & 0.873 & $0.723(0.016)$ & $0.786(0.003)$ \\
\hline \multirow{4}{*}{$\begin{array}{c}\text { refined } \\
\text { KIBA } \\
\text { Dataset }\end{array}$} & DeepDTA & $0.892(0.026)$ & 0.152 & 0.896 & $0.766(0.085)$ & $0.798(0.063)$ \\
\hline & $\begin{array}{l}\text { Attention- } \\
\text { DTA }\end{array}$ & $0.880(0.001)$ & 0.158 & 0.883 & $0.742(0.015)$ & $0.795(0.003)$ \\
\hline & $\begin{array}{l}\text { ELECTRA- } \\
\text { DTA- } \\
\text { LAST }\end{array}$ & $0.884(0.001)$ & 0.189 & 0.860 & $0.710(0.152)$ & $0.790(0.103)$ \\
\hline & $\begin{array}{l}\text { ELECTRA- } \\
\text { DTA-AVG }\end{array}$ & $0.894(0.001)$ & 0.148 & 0.890 & $0.769(0.018)$ & $0.802(0.010)$ \\
\hline
\end{tabular}

Table 6 Comparison of all baseline approaches and ELECTRA-DTA on the Davis datasets

\begin{tabular}{|c|c|c|c|c|c|c|}
\hline Dataset & Model & $\mathrm{Cl}$ & MSE & $\mathrm{R}$ & $r_{m}^{2}$ & AUPR \\
\hline \multirow{7}{*}{$\begin{array}{c}\text { Original } \\
\text { Davis } \\
\text { Dataset }\end{array}$} & KronRLS & 0.871 & 0.379 & - & 0.407 & 0.661 \\
\hline & SimBoost & 0.872 & 0.282 & - & 0.644 & 0.709 \\
\hline & DeepDTA & $0.876(0.004)$ & 0.261 & 0.846 & $0.630(0.017)$ & $0.714(0.010)$ \\
\hline & WideDTA & 0.886 & 0.262 & - & - & \\
\hline & DeepCDA & $0.891(0.003)$ & 0.248 & 0.857 & $0.649(0.009)$ & $0.739(0.006)$ \\
\hline & $\begin{array}{l}\text { ELECTRA- } \\
\text { DTA-AVG }\end{array}$ & $0.897(0.003)$ & 0.238 & 0.844 & $0.671(0.032)$ & $0.698(0.010)$ \\
\hline & $\begin{array}{l}\text { ELECTRA- } \\
\text { DTA- } \\
\text { LAST }\end{array}$ & $0.896(0.003)$ & 0.233 & 0.846 & $0.663(0.022)$ & $0.709(0.016)$ \\
\hline \multirow{4}{*}{$\begin{array}{c}\text { refined } \\
\text { Davis } \\
\text { Dataset }\end{array}$} & DeepDTA & $0.882(0.016)$ & 0.191 & 0.843 & $0.690(0.035)$ & $0.695(0.03)$ \\
\hline & $\begin{array}{l}\text { Attention- } \\
\text { DTA }\end{array}$ & $0.888(0.007)$ & 0.195 & 0.836 & $0.697(0.005)$ & $0.677(0.022)$ \\
\hline & $\begin{array}{l}\text { ELECTRA- } \\
\text { DTA- } \\
\text { LAST }\end{array}$ & $0.901(0.004)$ & 0.194 & 0.841 & $0.658(0.039)$ & $0.685(0.02)$ \\
\hline & $\begin{array}{l}\text { ELECTRA- } \\
\text { DTA-AVG }\end{array}$ & $0.902(0.003)$ & 0.198 & 0.842 & $0.637(0.048)$ & $0.686(0.039)$ \\
\hline
\end{tabular}


Table 7 Comparison of all baseline approaches and the ELECTRA-DTA on the BindingDB Dataset

\begin{tabular}{|c|c|c|c|c|c|c|}
\hline Dataset & Model & $\mathrm{Cl}$ & MSE & $\mathrm{R}$ & $r_{m}^{2}$ & AUPR \\
\hline \multirow{4}{*}{$\begin{array}{c}\text { Original } \\
\text { BindingDB } \\
\text { Dataset }\end{array}$} & DeepDTA & $0.812(0.002)$ & 0.832 & 0.824 & $0.623(0.02)$ & $0.443(0.01)$ \\
\hline & DeepCDA & $0.822(0.001)$ & 0.844 & 0.808 & $0.631(0.002)$ & $0.459(0.003)$ \\
\hline & $\begin{array}{l}\text { ELECTRA- } \\
\text { DTA-AVG }\end{array}$ & $0.832(0.004)$ & 0.852 & 0.693 & $0.645(0.012)$ & $0.807(0.002)$ \\
\hline & $\begin{array}{l}\text { ELECTRA- } \\
\text { DTA- } \\
\text { LAST }\end{array}$ & $0.829(0.001)$ & 0.848 & 0.707 & $0.653(0.023)$ & $0.801(0.002)$ \\
\hline \multirow{4}{*}{$\begin{array}{c}\text { refined } \\
\text { BindingDB } \\
\text { Dataset }\end{array}$} & DeepDTA & $0.826(0.001)$ & 0.703 & 0.845 & $0.669(0.004)$ & $0.795(0.003)$ \\
\hline & $\begin{array}{l}\text { Attention- } \\
\text { DTA }\end{array}$ & $0.804(0.003)$ & 0.844 & 0.811 & $0.619(0.009)$ & $0.764(0.004)$ \\
\hline & $\begin{array}{l}\text { ELECTRA- } \\
\text { DTA- } \\
\text { LAST }\end{array}$ & $0.838(0.001)$ & 0.861 & 0.641 & $0.690(0.02)$ & $0.812(0.002)$ \\
\hline & $\begin{array}{l}\text { ELECTRA- } \\
\text { DTA-AVG }\end{array}$ & $0.841(0.002)$ & 0.864 & 0.626 & $0.690(0.024)$ & $0.816(0.003)$ \\
\hline
\end{tabular}

For the original KIBA dataset, the proposed ELECTRA-DTA-AVG and DeepCDA achieve the best performance metrics, with ELECTRA-DTA-AVG outperforming DeepCDA by $0.016,0.025$ and 0.063 for MSE, R, and the $r_{m}^{2}$ index, respectively, while DeepCDA achieved the best CI (0.889 vs 0.885 ) and AUPR curve score. Both models achieved much better prediction performance than DeepDTA, SimBoost and KronRLS. As seen from Table 5, the proposed ELECTRA-DTA-AVG achieved values of $0.894,0.148,0.890,0.769$, and 0.802 for CI, MSE, R, $r_{m}^{2}$ index and AUPR curve score, respectively, for the refined KIBA dataset. Except for the $\mathrm{R}$ metric, our proposed method outperformed DeepDTA and AttentionDTA. With respect to the MSE metric, the proposed ELECTRA-DTA-LAST performed the worst, with a value of 0.189 , among all models.

For the original Davis dataset as in table 6, ELECTRA-DTA-AVG outperformed the next-best model in terms of the $\mathrm{CI}$ and $r_{m}^{2}$ index by 0.006 and 0.035 , respectively. Additionally, the MSE error was 0.238 , which was lower than that of the state-ofthe-art methods. It should also be noted that DeepCDA performed better than ELECTRA-DTA in terms of the AUPR curve score. For the refined Davis dataset, the proposed ELECTRA-DTA-AVG and ELECTRA-DTA-LAST models had the highest CIs (demonstrating improvements of 0.014 and 0.013 , respectively). Our methods did not generate the best results for the other metrics, but the differences were small.

Table 7 summarizes the evaluation metrics of the various methods for the original and refined BindingDB datasets. ELECTRA-DTA-AVG outperformed the baseline approaches for all evaluation metrics. For the original dataset, ELECTRA-DTAAVG obtained a 0.007 increase in the CI, while the MSE value of our method was 0.109 lower than that of DeepCDA. The most substantial improvement was observed for the AUPR curve score, which increased from 0.459 for DeepCDA to 0.829. For the refined BindingDB dataset, our two ELECTRA-based models exceeded DeepDTA and AttentionDTA for all evaluation metrics, with ELECTRA-DTA-AVG yielding the best performance. Among all evaluation metrics, MSE has the most noticeable difference, which decreasing from 0.844 for AttentionDTA to 0.626. The improvement in the CI was distinct, achieving the highest value (0.841) over the baselines. The $r_{m}^{2}$ index, $\mathrm{R}$ and the AUPR curve score improved substantially relative to those of AttentionDTA and DeepDTA. ELECTRA-DTA-LAST suffered from a higher MSE error than ELECTRA-DTA-AVG. This higher MSE indicates 
that the use of the only last embedding layer of ELECTRA is insufficient for ob- ${ }^{359}$ taining a more accurate representation for difficult datasets. It should be noted that ${ }_{360}$ ELECTRA-DTA achieved better results in the BindingDB dataset in all metrics 361 than the baseline models, likely due to differences in the dataset distribution. Ac- 362 cording to an analysis of these datasets, the BindingDB dataset has sparser samples, $\quad 363$ thereby making it more difficult to train prediction models. This suggests that our 364 model has more robustness and reliability.

Interestingly, we observed that the CIs for DeepDTA and ELECTRA-DTA in- 366 creased significantly from the original to the refined datasets. The reason for this 367 is that different proteins or drugs have identical representations in the original ${ }_{368}$ datasets, and consequently, in the deep learning-based methods, the network be- ${ }_{369}$ comes confuses as to how to learn an effective representation for these proteins or 370 drugs.

According to the reported results in Tables 5, 6 and 7, ELECTRA-DTA and DeepCDA consistently performed the other DTA models in terms of all metrics, with ELECTRA-DTA-AVG being responsible for 10 of the 15 best scores for the refined datasets, indicating that our model has more reliable and accurate predictive performance than the other models. This experiment also indicated that the performance of ELECTRA-DTA-AVG is superior to that of ELECTRA-DTA-LAST, especially in terms of the CI, over all refined datasets. This shows that the output from each layer in ELECTRA is useful in solving the DTA problem.

Results of predictive performance of warm/cold splitting settings the discovery of the binding affinity for unseen drugs or targets. Therefore, in these ${ }_{382}$ experiments, three splitting schemes were used for all refined datasets: 383

1 Warm splitting: Every drug or target in the test set is encountered in the ${ }_{384}$ training set.

2 Cold-drug splitting: Every drug in the test set is absent in the training set. 386

3 Cold-target splitting: Every target in the test set is absent from the training 387 set.

4 Blind splitting: the target and the drug are both absent in the training set. ${ }_{389}$ The cold-drug, cold-target and blinding splits deliver realistic and more difficult 390 appraisal schemes for the DTA problem. For these splitting schemes, the targets 391 and proteins are both split at an 8/2 ratio for the training/testing set. 392

Figure 6 reports a seemingly contradictory phenomenon: all methods perform ${ }_{393}$ better in the cold-target setting with the Davis dataset but worse with the KIBA 394 dataset than in the cold-drug setting. We see that the prediction performance of the 395 deep learning-based methods is correlated to the data distribution of the dataset. 396 Combined with the data in Table 4, it can be seen that when the number of drugs ${ }^{397}$ in the training set is large and the number of targets is small, the cold-target 398 splitting scheme tends to yield a more challenging problem than the cold-drug 399 splitting scheme; on the other hand, when there are many targets and few drugs, 400 the cold-target predictions appear to be better than the cold-drug predictions. We ${ }_{401}$ hypothesize that the reason behind such contradictory results could be that the ${ }^{402}$ models become more stable with greater knowledge on entities (drugs or targets) ${ }_{403}$ 
in the training sets. In addition, we also observe an interesting phenomenon in ${ }_{404}$ which the gap between the cold-drug and cold-target predictions is different over ${ }_{405}$ the KIBA and Davis datasets. The cold-drug and cold-target prediction CIs are ${ }_{406}$ similar in the KIBA dataset, even though it has 2052 drugs and only 229 targets. ${ }_{407}$ Along this line, one might conclude that the model needs many more types of drugs ${ }_{408}$ than targets to learn their chemical representations. As expected, the performance 409 of the warm splitting scheme tends to be better than that of the cold-drug and ${ }_{410}$ cold-target splitting schemes.

Additionally, we realized that the blinding split schemes that had fewer drug- ${ }_{412}$ target pairs turned out to be the most challenging for the deep learning based ${ }_{413}$ models. We can observed that all the models performance decreased drastically ${ }_{414}$ while our methods usually exhibited a relatively stable performance on the three ${ }_{415}$ datasets. We reckon that the reason is due the proposed pretrained ELECTRA ${ }_{416}$ model can extract the abundant information.

Case Study on Drug Repurposing for COVID-19

We conducted a case study to showcase the use of our proposed ELECTRA-DTA ${ }_{419}$ for biomedical researchers for the repurposing of existing drugs. Here, we took key ${ }_{420}$ proteins from SARS-CoV-2 as our targets and selected potential drugs from among ${ }_{421}$ the 82 known antiviral drugs approved or in development for treating infections ${ }_{422}$ caused by HIV, hepatitis B virus (HBV), hepatitis C virus (HCV) and influenza [24]. ${ }_{423}^{42}$ SARS-CoV-2 is an enveloped, positive-sense, single-stranded RNA beta-coronavirus ${ }_{424}^{4}$ [25] with a similar structure to the severe acute respiratory syndrome coronavirus ${ }_{425}$ (SARS-CoV) and Middle East respiratory syndrome coronavirus (MERS-CoV). ${ }_{426}$ Three categories of proteins are encoded by the SARS-CoV-2 genome, including ${ }_{427}$ non-structural proteins (such as 3-chymotrypsin-like protease (3CL Pro), papain- ${ }_{428}$ like protease, helicase, and RNA-dependent RNA polymerase (RdRp)), structural ${ }_{429}$ proteins (such as spike glycoprotein) and accessory proteins. The four non-structural 430 proteins and the spike glycoprotein mentioned above are essential for the viral life ${ }_{431}$ cycle and virus-cell receptor interactions; consequently, these five proteins were rec- ${ }_{432}$ ognized as attractive targets. Motivated by this knowledge, we used 3CL Pro and ${ }_{433}$ RdRp as examples and then applied the ELECTRA-DTA model trained on the ${ }_{434}$ BindingDB database to report the binding affinity scores of each drug candidate ${ }_{435}$ and targeted protein. Drug candidates were ranked according to their predicted ${ }_{436}$ binding affinity scores with 3CL Pro and Rdpd (as shown in supplementary table ${ }_{437}$ S1). Additionally, the top 10 drugs with the highest binding affinity for 3CL Pro or ${ }_{438}$ Rdpd are also provided, for a total of 14 drugs, as listed in Table 8.

Among these, saquinavir, an antiviral protease inhibitor, was predicted to have ${ }_{440}$ the highest binding affinity with 3CL Pro with ELECTRA-DTA. In Singapore, ${ }_{441}$ saquinavir has been used to treat patients with SARS-CoV-2. When Rdpd was ${ }_{442}$ used as the target, daclatasvir had the strongest binding affinity value. Some studies ${ }_{443}$ have supported daclatasvir as a potential drug for treating COVID-19. Darunavir ${ }_{444}$ and cobicistat were both among the top-ranked drugs. In an ongoing clinical trial in 445 China, darunavir and cobicistat were assessed for their efficacy and safety. Lopinavir $\quad{ }_{446}$ has been reported to inhibit SARS-CoV-2 at a half-maximum efficacy concentration $\quad{ }_{447}$ (EC50) - the level of a drug that induces a response halfway between baseline and ${ }_{448}$ 
maximum after a specified exposure time - of $26.36 \mu \mathrm{M}$. Lopinavir has also been ${ }^{449}$ listed as an emergency drug for COVID-19 [26]. Administration of lopinavir as ${ }_{450}$ an emergency drug in China was shown to increase the eosinophil count among ${ }_{451}$ COVID-19 patients [27].

Overall, there is promising evidence from the literature or clinical trials on $12{ }^{453}$ recommendations out of the 14 highest-affinity drugs, which illustrates the po- ${ }^{454}$ tential of ELECTRA-DTA for recommending high-quality repurposing candidates. ${ }^{455}$ Consequently, the abovementioned drugs could be regarded as candidates to treat ${ }_{456}$ COVID-19 infections, but further study on the efficiency of these drugs is also nec- ${ }^{457}$ essary. It should be noted that our ELECTRA-DTA model only uses target and ${ }_{458}$ drug sequence information without any details on current ongoing clinical trials. ${ }^{459}$ Information from clinical trials was only used to affirm the efficacy of our approach. $\quad{ }_{460}$

\section{Discussion}

This study presented ELECTRA-DTA, a DTA prediction approach with two trained ${ }_{462}$ ELECTRA models for encoding protein amino acid sequences and compound SMI- ${ }_{463}$ LESs separately in an unsupervised manner. For compound SMILES strings, all ${ }_{464}$ characters found in different SMILESs were treated as the vocabulary, and selected 465 compounds from the PubChem dataset were used as a corpus to obtain vector ${ }_{466}$ representations of each character, subsequently yielding a vector sequence for each ${ }_{467}$ compound SMILES. The unsupervised training on a large-scale sample space in- ${ }_{468}$ corporated additional contextual information, including potential substructures or $\quad 469$ function groups. The ELECTRA-based embedding mechanism and the design of our $\quad 470$ network architecture ensured a better predictive ability than that of state-of-the-art ${ }_{471}$ baseline methods; moreover, they provide a feasible encoding module that can be $\quad 472$ stacked over other feature representation networks as the embedding layer for pro- $\quad 473$ tein sequences or compound SMILESs in various learning tasks. In addition, they ${ }_{474}$ can also effectively train a new embedding model by constructing domain-specific $\quad 475$ corpora for specific downstream tasks.

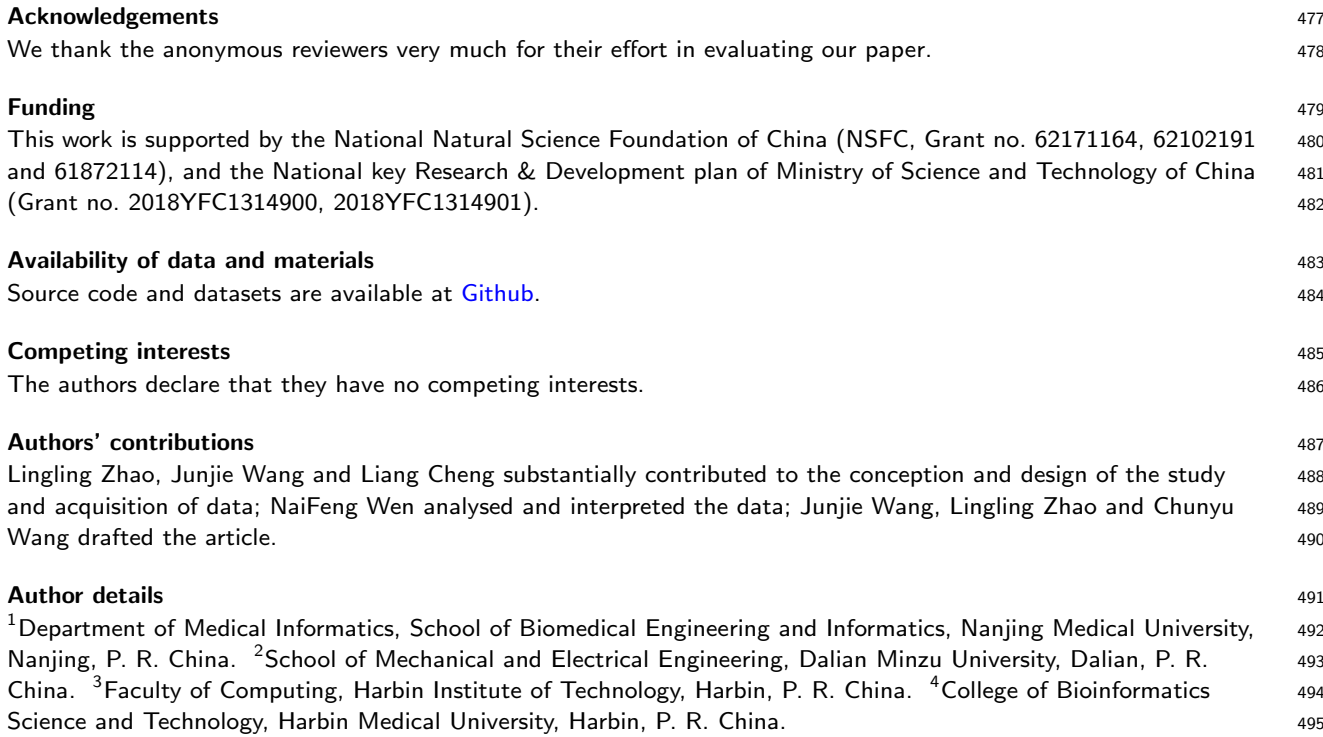


References

1. He, T., Heidemeyer, M., Ban, F., Cherkasov, A., Ester, M.: Simboost: a read-across approach for predicting drug-target binding affinities using gradient boosting machines. Journal of Cheminformatics 9(1), 24-24 (2017)

2. Pahikkala, T., Airola, A., Pietilä, S., Shakyawar, S., Szwajda, A., Tang, J., Aittokallio, T.: Toward more realistic drug-target interaction predictions. Briefings in bioinformatics 16(2), 325-337 (2015)

3. Öztürk, H., Özgür, A., Ozkirimli, E.: Deepdta: deep drug-target binding affinity prediction. Bioinformatics 34(17), 821-829 (2018)

4. Karimi, M., Wu, D., Wang, Z., Shen, Y.: Deepaffinity: interpretable deep learning of compound-protein affinity through unified recurrent and convolutional neural networks. Bioinformatics 35(18), 3329-3338 (2019)

5. Sutskever, I., Vinyals, O., Le, Q.V.: Sequence to sequence learning with neural networks. In: Advances in Neural Information Processing Systems 27, vol. 27, pp. 3104-3112 (2014)

6. Devlin, J., Chang, M.-W., Lee, K., Toutanova, K.N.: Bert: Pre-training of deep bidirectional transformers for language understanding. In: Proceedings of the 2019 Conference of the North American Chapter of the Association for Computational Linguistics: Human Language Technologies, Volume 1 (Long and Short Papers) pp. 4171-4186 (2018)

7. Clark, K., Luong, M.-T., Le, Q.V., Manning, C.D.: Electra: Pre-training text encoders as discriminators rather than generators. In: ICLR 2020 : Eighth International Conference on Learning Representations (2020)

8. Bolton, E.E., Wang, Y., Thiessen, P.A., Bryant, S.H.: Pubchem: Integrated platform of small molecules and biological activities. vol. 4, pp. 217-241 (2008)

9. Kim, S., Thiessen, P.A., Bolton, E.E., Chen, J., Fu, G., Gindulyte, A., Han, L., He, J., He, S., Shoemaker, B.A., Wang, J., Yu, B., Zhang, J.-J., Bryant, S.H.: Pubchem substance and compound databases. Nucleic Acids Research 44, 1202-1213 (2016)

10. Kim, S., Chen, J., Cheng, T., Gindulyte, A., He, J., He, S., Li, Q., Shoemaker, B.A., Thiessen, P.A., Yu, B. Zaslavsky, L., Zhang, J., Bolton, E.E.: Pubchem 2019 update: improved access to chemical data. Nucleic Acids Research 47 (2019)

11. O'Boyle, N.M., Banck, M., James, C.A., Morley, C., Vandermeersch, T., Hutchison, G.R.: Open babel: An open chemical toolbox. Journal of Cheminformatics 3(1), 33-33 (2011)

12. Bateman, A., Martin, M.J., O’Donovan, C., Magrane, M., Apweiler, R., Alpi, E., Antunes, R., Arganiska, J., Bely, B., Bingley, M., Bonilla, C., Britto, R., Bursteinas, B., Chavali, G., Cibrian-Uhalte, E., Silva, A.D., Giorgi, M.D., Dogan, T., Fazzini, F., Gane, P., Castro, L.G., Garmiri, P., Hatton-Ellis, E., Hieta, R., Huntley, R., Legge, D., Liu, W., Luo, J., Macdougall, A., Mutowo, P., Nightingale, A., Orchard, S., Pichler, K., Poggioli, D., Pundir, S., Pureza, L., Qi, G., Rosanoff, S., Saidi, R., Sawford, T., Shypitsyna, A., Turner, E., Volynkin, V., Wardell, T., Watkins, X., Zellner, H., Cowley, A., Figueira, L., Li, W., McWilliam, H., Lopez, R., Xenarios, I., Bougueleret, L., Bridge, A., Poux, S., Redaschi, N., Aimo, L., Argoud-Puy, G., Auchincloss, A., Axelsen, K., Bansal, P., Baratin, D., Blatter, M.C., Boeckmann, B., Bolleman, J., Boutet, E., Breuza, L., Casal-Casas, C., Castro, E.D., Coudert, E., Cuche, B., Doche, M., Dornevil, D., Duvaud, S., Estreicher, A., Famiglietti, L., Feuermann, M., Gasteiger, E., Gehant, S., Gerritsen, V., Gos, A., Gruaz-Gumowski, N., Hinz, U., Hulo, C., Jungo, F., Keller, G., Lara, V., Lemercier, P., Lieberherr, D., Lombardot, T., Martin, X., Masson, P., Morgat, A., Neto, T., Nouspikel, N., Paesano, S., Pedruzzi, I., Pilbout, S., Pozzato, M., Pruess, M., Rivoire, C., Roechert, B., Schneider, M., Sigrist, C., Sonesson, K., Staehli, S., Stutz, A., Sundaram, S., Tognolli, M., Verbregue, L., Veuthey, A.L., Wu, C.H., Arighi, C.N., Arminski, L., Chen, C., Chen, Y., Garavelli, J.S., Huang, H., Laiho, K., McGarvey, P., Natale, D.A., Suzek, B.E., Vinayaka, C.R., Wang, Q., Wang, Y., Yeh, L.S., Yerramalla, M.S., Zhang, J.: Uniprot: A hub for protein information. Nucleic Acids Research 43 (2015)

13. Rohanian, O., Taslimipoor, S., Kouchaki, S., Ha, L.A., Mitkov, R.: Bridging the gap: Attending to discontinuity in identification of multiword expressions. In: Proceedings of the 2019 Conference of the North American Chapter of the Association for Computational Linguistics: Human Language Technologies, Volume 1 (Long and Short Papers), pp. 2692-2698 (2019)

14. Tang, J., Szwajda, A., Shakyawar, S., Xu, T., Hintsanen, P., Wennerberg, K., Aittokallio, T.: Making sense of large-scale kinase inhibitor bioactivity data sets: a comparative and integrative analysis. Journal of Chemical Information and Modeling 54(3), 735-743 (2014)

15. Davis, M.I., Hunt, J.P., Herrgard, S., Ciceri, P., Wodicka, L.M., Pallares, G., Hocker, M., Treiber, D.K., Zarrinkar, P.P.: Comprehensive analysis of kinase inhibitor selectivity. Nature Biotechnology 29(11), 1046-1051 (2011)

16. Liu, T., Lin, Y., Wen, X., Jorissen, R.N., Gilson, M.K.: Bindingdb: a web-accessible database of experimentally determined protein-ligand binding affinities. Nucleic Acids Research 35, 198-201 (2007)

17. Tybulewicz, V.L.J., Crawford, C.E., Jackson, P.K., Bronson, R.T., Mulligan, R.C.: Neonatal lethality and lymphopenia in mice with a homozygous disruption of the c-abl proto-oncogene. Cell 65(7), 1153-1163 (1991)

18. Cristiani, C., Rusconi, L., Perego, R., Schiering, N., Kalisz, H.M., Knapp, S., Isacchi, A.: Regulation of the wild-type and y1235d mutant met kinase activation. Biochemistry 44(43), 14110-14119 (2005)

19. Gönen, M., Heller, G.: Concordance probability and discriminatory power in proportional hazards regression. Biometrika 92(4), 965-970 (2005)

20. Pratim Roy, P., Paul, S., Mitra, I., Roy, K.: On two novel parameters for validation of predictive qsar models. Molecules 14(5), 1660-1701 (2009)

21. Roy, K., Chakraborty, P., Mitra, I., Ojha, P.K., Kar, S., Das, R.N.: Some case studies on application of "rm2" metrics for judging quality of quantitative structure-activity relationship predictions: emphasis on scaling of response data. Journal of computational chemistry 34(12), 1071-1082 (2013)

22. Abbasi, K., Razzaghi, P., Poso, A., Amanlou, M., Ghasemi, J.B., Masoudi-Nejad, A.: Deepcda: Deep cross-domain compound-protein affinity prediction through Istm and convolutional neural networks. Bioinformatics 36(17), 4633-4642 (2020)

23. Zhao, Q., Xiao, F., Yang, M., Li, Y., Wang, J.: Attentiondta: prediction of drug-target binding affinity using attention model. In: 2019 IEEE International Conference on Bioinformatics and Biomedicine (BIBM), pp. 64-69 
(2019). IEEE

567

24. Hosseini, F.S., Amanlou, M.: Anti-hcv and anti-malaria agent, potential candidates to repurpose for coronavirus 568 infection: Virtual screening, molecular docking, and molecular dynamics simulation study. Life sciences $\mathbf{2 5 8}$, $118205(2020)$

25. Pant, S., Singh, M., Ravichandiran, V., Murty, U., Srivastava, H.K.: Peptide-like and small-molecule inhibitors against covid-19. Journal of Biomolecular Structure and Dynamics, 1-10 (2020)

26. Hu, F., Jiang, J., Yin, P.: Prediction of potential commercially inhibitors against sars-cov-2 by multi-task deep model. arXiv preprint arXiv:2003.00728 (2020)

27. Feng, Z Chen, M. Xue, Y Liang, T., Chen, $H$, Zhou, Y Nolin, T.D Smith, R B., Xie, X-Q. Mccs: a novel recognition pattern-based method for fast track discovery of anti-sars-cov-2 drugs. Briefings in 576 bioinformatics 22(2), 946-962 (2021)

\section{Additional Files}

Additional file 1 - table 8.docx

Ranked drug candidates for the $3 \mathrm{CL}$ protease and Rdpd proteins of SARS-CoV-2 with predicted affinity.

Additional file 2 - duplicate-davis.txt

The removed samples for the Davis Dataset.

Additional file 3 - duplicate-kiba.txt

The removed samples for the KIBA Dataset.

Additional file 3 - duplicate-bindingdb.txt 
Figures

Step 0: Embedding Model Pretraining
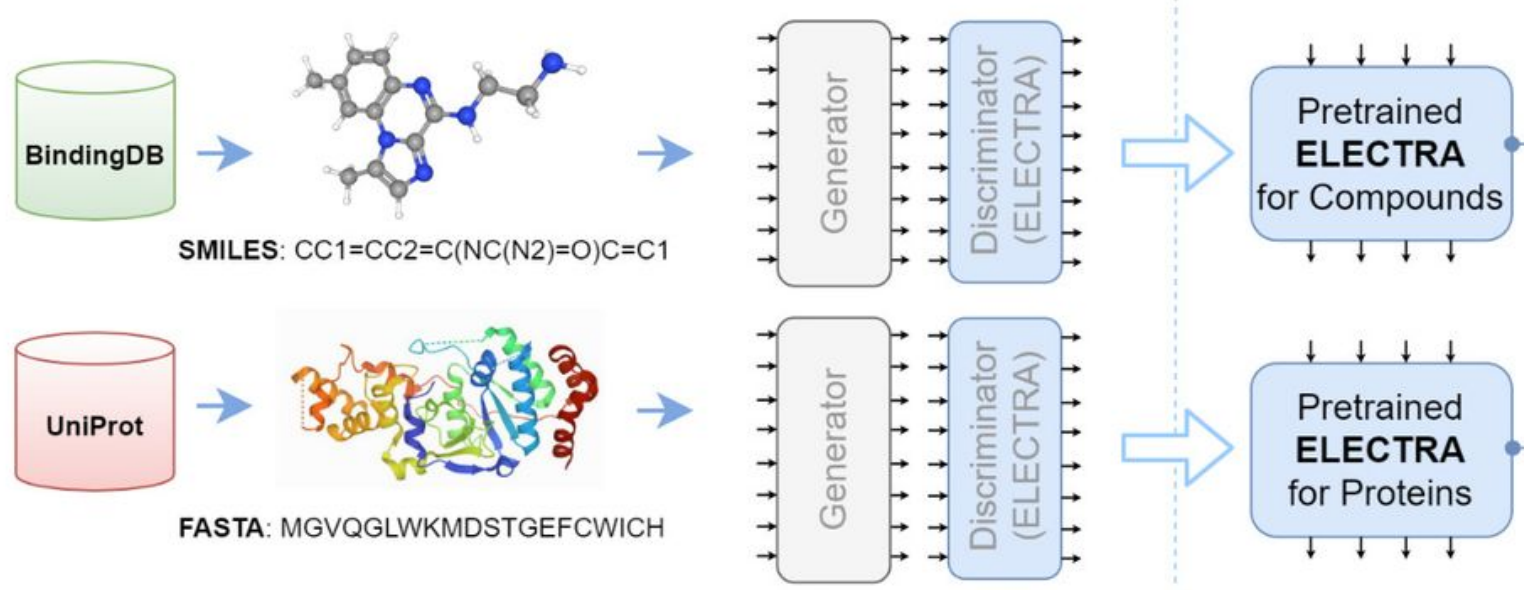

Step 1: Compound and Protein Representation

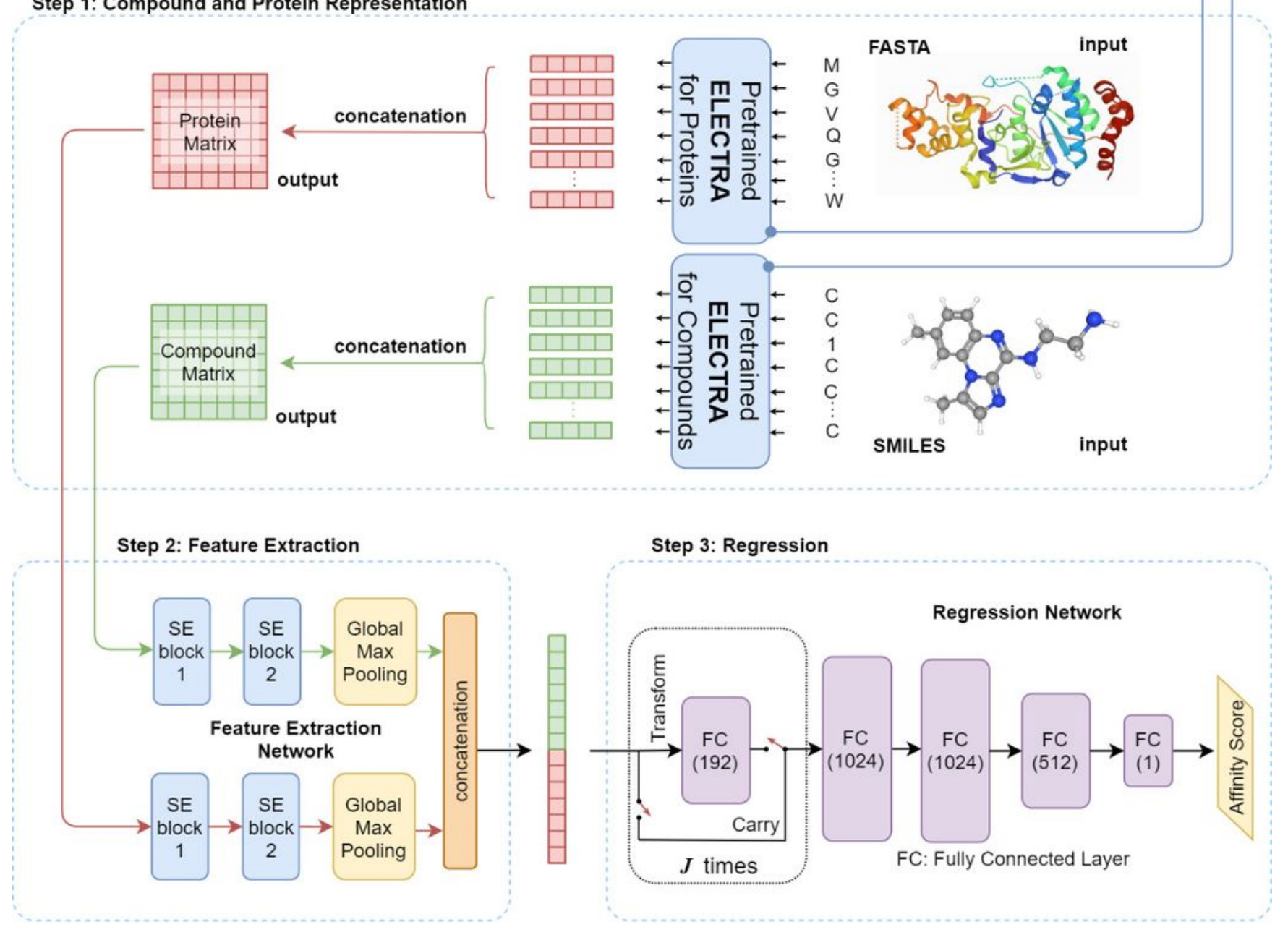

Figure 1

Overview of ELECTRA-DTA 


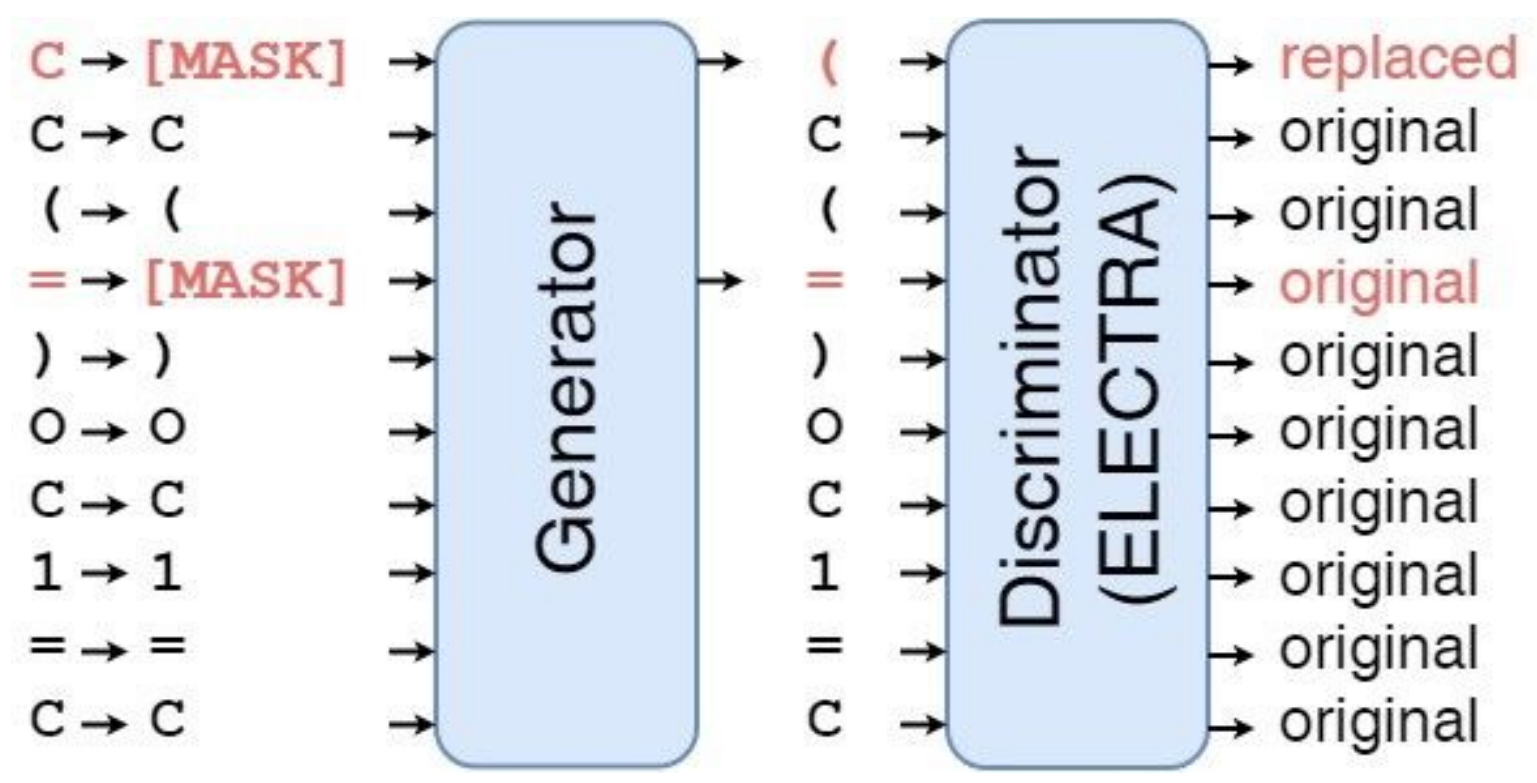

Figure 2

The principle of ELECTRA pre-training

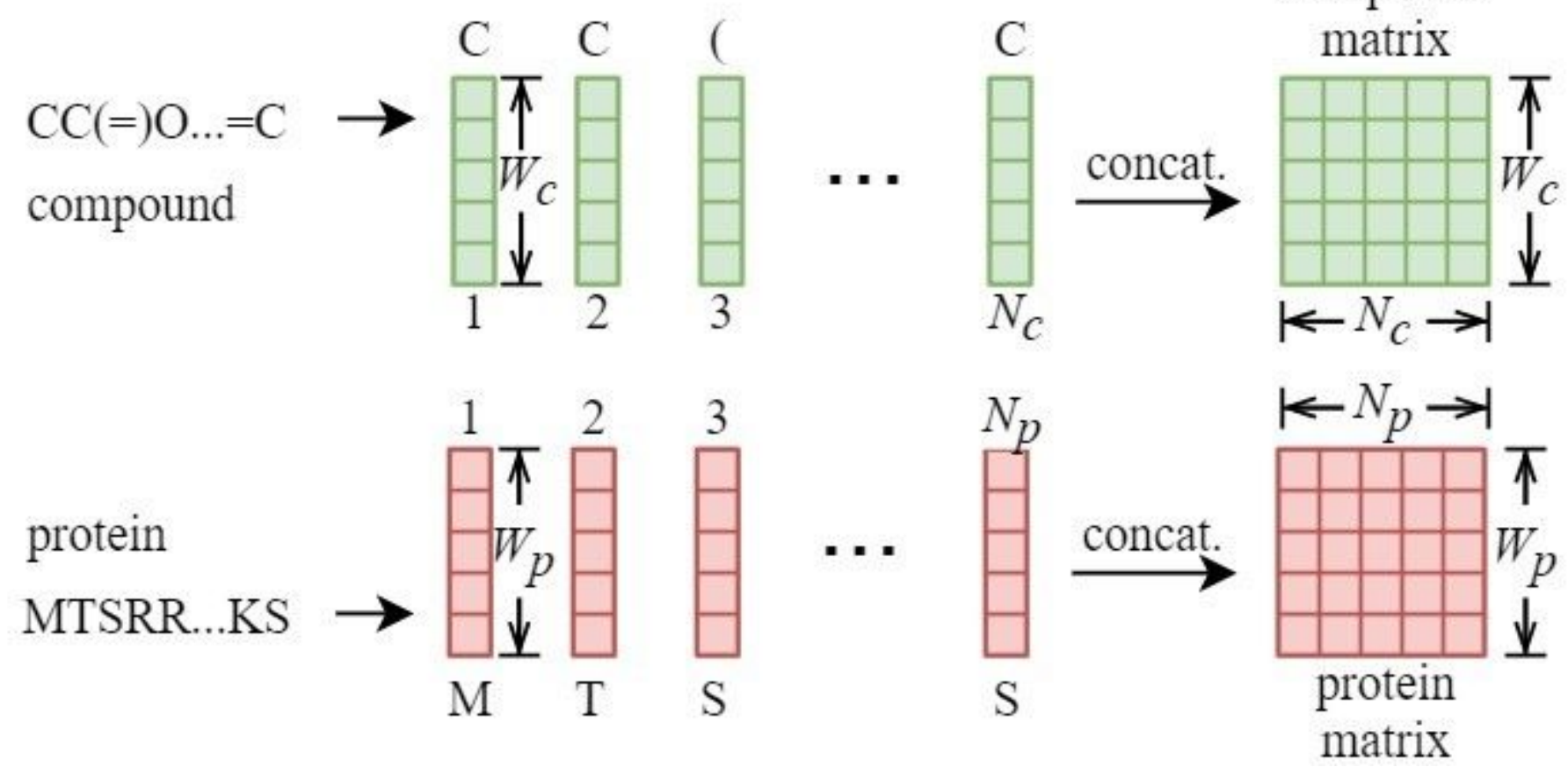

Figure 3

Compound SMILES and protein sequence embedding 


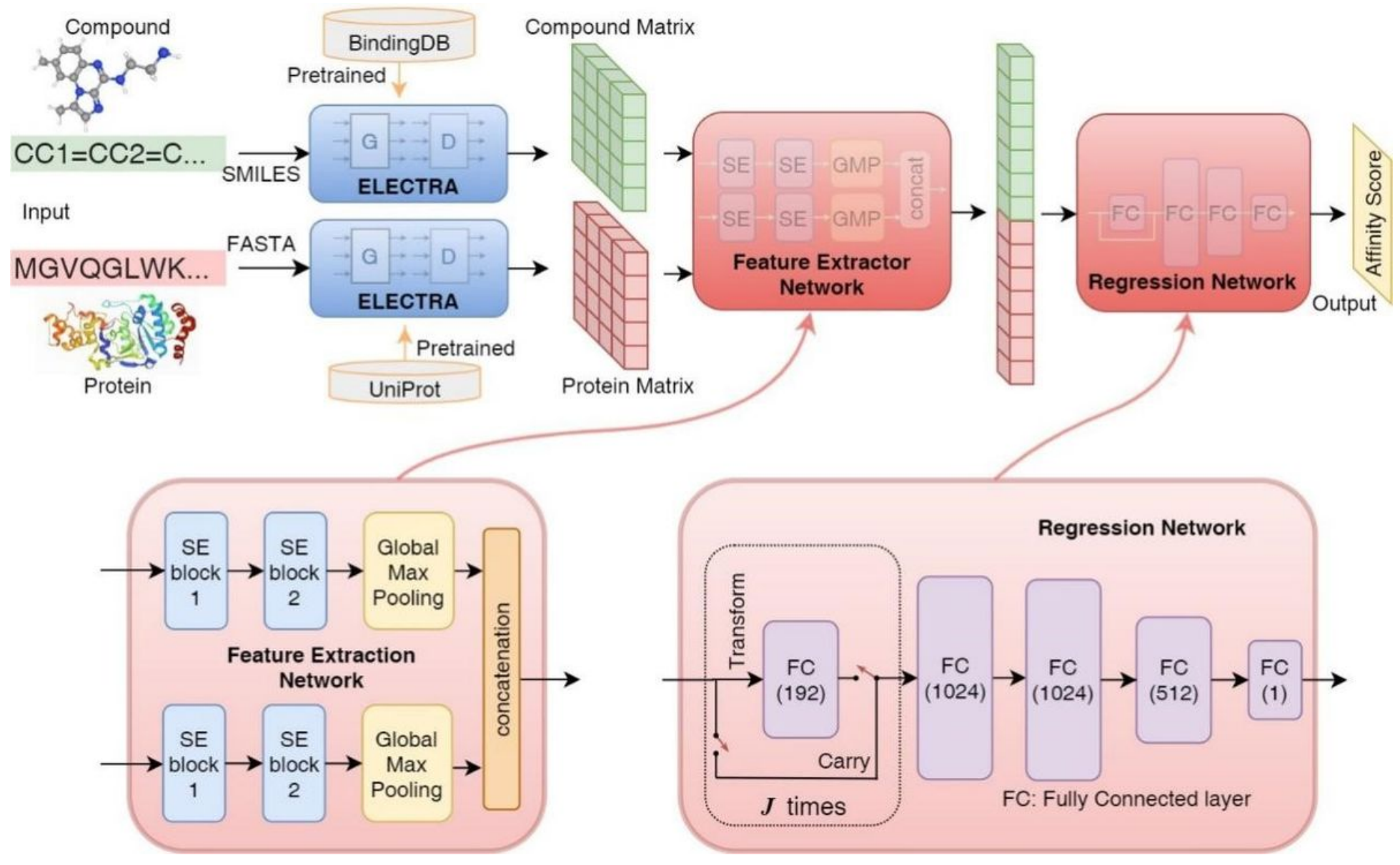

Figure 4

Architecture of the ELECTRA-DTA model

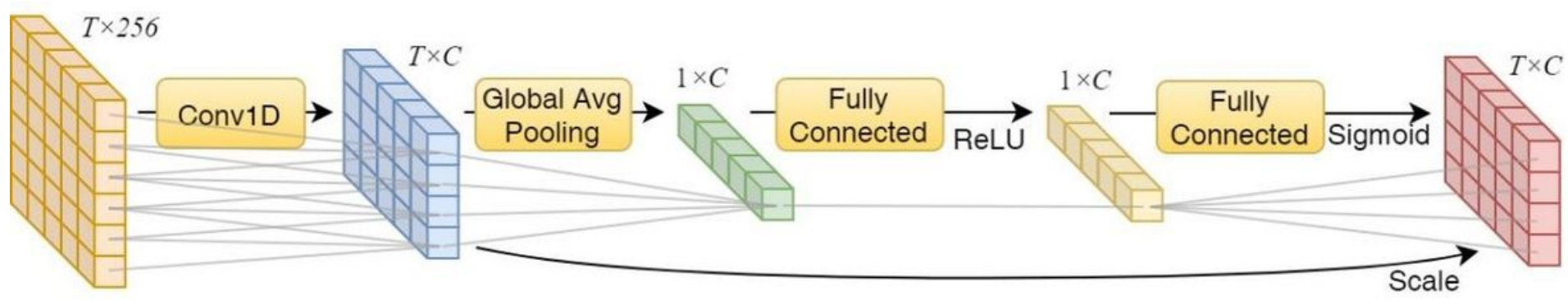

Figure 5

Structure of the squeeze-and-excitation block 

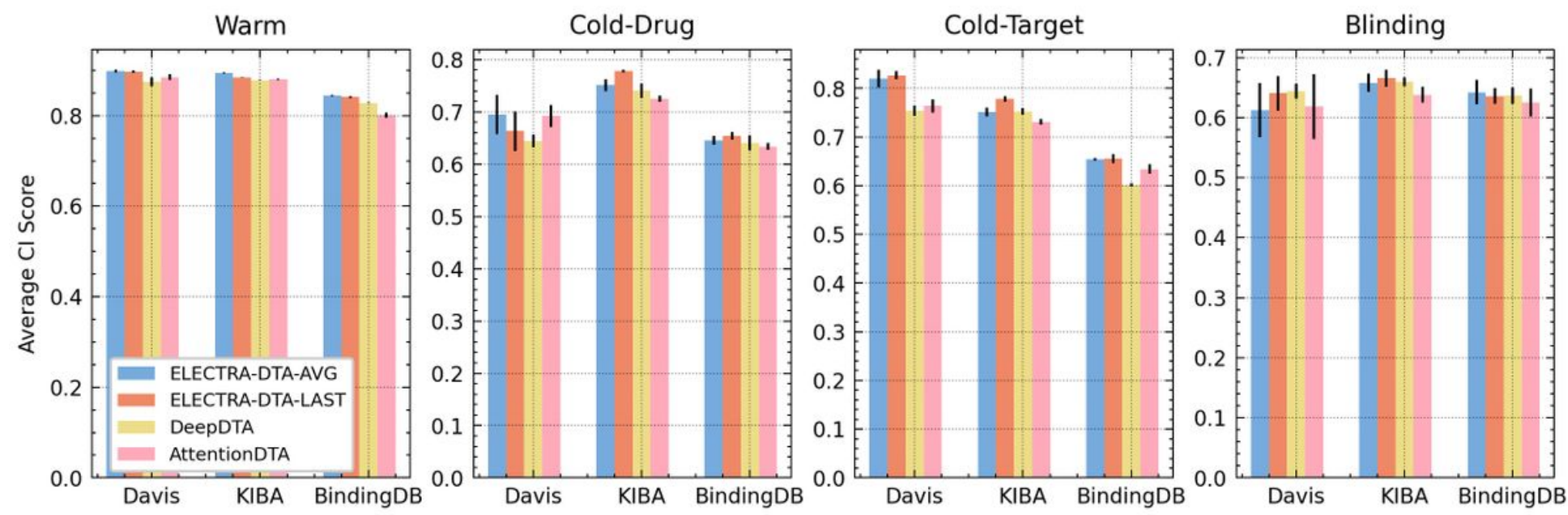

Figure 6

Figure 6 reports a seemingly contradictory phenomenon

\section{Supplementary Files}

This is a list of supplementary files associated with this preprint. Click to download.

- duplicatedavis.txt

- duplicatekiba.txt

- duplicatebindingdb.txt 